\title{
MODELAGEM PARA DESCRIÇÃO DA CINÉTICA DE SECAGEM DE FOLHAS DE PARIRI (Arrabidaea chica (Humb. \& Bonpl) B. Verlot) POR INFRAVERMELHO E SECADOR DE BANDEJAS.
}

\author{
M. M. FERREIRA ${ }^{1}$, R. N. CUNHA ${ }^{1}$ \\ ${ }^{1}$ Centro Universitário de Patos de Minas, Departamento de Engenharia Química \\ E-mail para contato: mariana.academico@gmail.com
}

\begin{abstract}
RESUMO - As folhas da espécie Arrabidaea chica (Humb. \& Bonpl) B. Verlot, conhecida como Pariri vêm sendo utilizadas na cosmetologia devido ao efeito adstringente de seu extrato. $\mathrm{O}$ estudo fitoquímico de plantas medicinais se baseia na secagem prévia das folhas. Objetivou-se, neste estudo, determinar e modelar as curvas de secagem das folhas de Pariri por infravermelho e secador de bandejas. Faz ainda parte do escopo a avaliação da influência da temperatura sobre o coeficiente de difusão efetivo. As secagens foram conduzidas nas temperaturas de $50,60,65$, e $70^{\circ} \mathrm{C}$. Aos dados experimentais foram ajustados modelos matemáticos, cujas magnitudes do coeficiente de determinação $\left(\mathrm{R}^{2}\right)$ foram utilizadas para verificar o grau de ajuste dos modelos. Do estudo concluiu-se que: a) o comportamento das curvas de secagem das folhas de Pariri foi semelhante ao da maioria dos produtos agrícolas; b) o modelo matemático de secagem proposto por Midilli et al. foi o que melhor se ajustou aos dados experimentais; c) qualitativamente verifica-se que a cor das folhas de Pariri sofreu alterações devido à temperatura de secagem tornando-se mais escuras devido à perda de clorofila.
\end{abstract}

\section{INTRODUÇÃO}

A espécie Arrabidaea chica (Humb. \& Bonpl) B. Verlot, encontrada principalmente na Região Amazônica, é popularmente conhecida como Crajiru, Carajiru, Pariri e Chica, cuja espécie pertence à família Bignoniaceae. O Brasil é, provavelmente, a região onde a família apresenta-se com o maior número de espécies, ocorrendo desde a Amazônia até o Rio Grande do Sul (Pauletti et al., 2003).

As folhas da espécie são utilizadas popularmente na forma de chás para o tratamento de cólicas intestinais, anemias, inflamações uterinas, hemorragias, icterícia, albuminúria, micoses e lavagem de ferimentos na pele (Corrêa, 2004).

O primeiro estudo fitoquímico das folhas de Pariri (Chapman et al., 1927) citado por (Taffarello,2008) relata o isolamento de 3-deoxiantocianidina (Carajurina). Posteriormente, Harborne e Willians (1998) propuseram que a ocorrência deste raro pigmento em Bignoniaceae era provavelmente restrita a essa planta. Taffarello (2008) mostra também em seus estudos com Pariri, o resultado de testes positivos para antraquinonas, esteroides, triterpenos, flavonoides, saponinas e taninos. 
A secagem de material vegetal tem como objetivo cessar as alterações químicas dos tecidos e evitar possíveis degradações durante o seu armazenamento, mantendo, assim, suas características físicas e químicas por mais tempo (Corrêa et al., 2004). Sendo assim, a secagem de plantas medicinais contribui para o armazenamento seguro, garantindo-lhes a permanência de suas propriedades medicinais, visando atender às necessidades das indústrias farmacêuticas de fitoterápicos, os quais não têm meios de utilizar plantas frescas na quantidade exigida para a produção industrial (Lorenzi e Matos, 2008 citado por Junqueira, 2016).

A tecnologia de secagem via radiação infravermelha, tem sido amplamente utilizada em vários processos industriais, envolvendo materiais têxteis, eletrônicos, tintas automotivas e papéis. A radiação infravermelha pode apresentar vantagens em relação à secagem convectiva convencional, tais como: simplicidade do equipamento, fácil acoplamento do aquecimento infravermelho com o aquecimento convectivo e condutivo, rápida resposta transiente e economia de energia (Ratti e Mujumdar, 1995 citado por Pereira, 2009). Um dos fatores para o uso de radiação eletromagnética no processo de secagem é a necessidade de se aplicar fontes alternativas de energia para reduzir o tempo e os custos com o processo (Salagnac et al., 2004). A secagem em secador de bandeja, a circulação de ar é feita por um ventilador situado atrás de resistências elétricas usadas para o aquecimento do ar de entrada, em que o controle da temperatura realizado por um termostato (Celestino, 2010).

A cinética de secagem é de fundamental importância para a modelagem matemática da operação e projeto de secadores. É através deste estudo que se estabelecem as equações da umidade em função do tempo de secagem para os diferentes períodos e taxas de secagem, possibilitando também a determinação do mecanismo predominante na transferência de massa do material para o fluído e as respectivas equações matemáticas correspondentes (Ferreira, 2004). Dentro desse contexto objetiva-se com este estudo avaliar as cinéticas de secagem de folhas de Pariri por infravermelho e secador de bandejas visando, em estudos posteriores, ponderar os efeitos da temperatura sobre os rendimentos dos extratos e os metabólitos secundários presentes na planta.

\section{MATERIAL E MÉTODOS}

\subsection{As folhas de Pariri}

Os experimentos foram conduzidos no Laboratório de Engenharia Química do Centro Universitário de Patos de Minas. As folhas de Pariri foram colhidas manualmente, sendo selecionadas apenas as folhas com ausência de partes danificadas.

\subsection{A cinética de secagem}

Foram adotadas as temperaturas de 50,60, 65 e $70^{\circ} \mathrm{C}$. Os estudos foram conduzidos em infravermelho (GEHAKA IV2500) com precisão de 0,001 g e secador de bandejas (PardalPS60). Durante os testes de infravermelho foram colocados, para secagem, aproximadamente 3,6 g de folhas de Pariri com pesagens em intervalos de $1 \mathrm{~min}$, até obtenção de massa constante. Após o término de cada tratamento de secagem as folhas secas foram acondicionadas em embalagens plásticas. As secagens em secadores de bandeja utilizaram-se as mesmas temperaturas, no entanto foram adicionadas 54,6 $\mathrm{g}$ de folhas e adotados intervalos de tempo entre as pesagens de 3 min. A umidade relativa do ar foi determinada através de 
termo-higrômetro, sendo essa constante em todos os ensaios experimentais (UR=45 $\pm 2 \%$ ). Em geral, tais experimentos fornecem dados de massa total do sólido úmido em diferentes instantes de tempo, possibilitando calcular a umidade total $X t$ e a umidade livre $X$, utilizando as Equações 1 e 2. Os dados experimentais foram expressos na forma de razão de umidade RU, conforme Equação 3, em que: $W$ é massa de sólido úmido [g] e Ws é massa de sólido seco [g].

$$
\begin{gathered}
X_{t}=\frac{W-W_{s}}{W_{s}} \\
X=X_{t}-X^{*} \\
R U=\frac{X_{t}-X^{*}}{X_{i}-X^{*}}
\end{gathered}
$$

Os valores de RU observados para cada temperatura do ar de secagem foram analisados pelas equações empíricas e semi-empírica (Page, Midilli et al., Henderson e Pabis e Newton e Lewis) de regressão não linear, utilizando-se o software Scilab 5.0. Ressalta-se que as constantes $a, b, n, k, k_{0}$ e $k_{1}$ são intrínsecas a cada modelo e que sendo o grau de ajuste dos modelos foi determinado por meio das magnitudes do coeficiente de determinação $\left(\mathrm{R}^{2}\right)$.

O coeficiente de difusão efetivo foi obtido por meio do ajuste do modelo matemático da difusão líquida, descrito pela Equação 4, aos dados experimentais da secagem das folhas de Pariri. A solução analítica para a segunda lei de Fick é apresentada na forma de uma série infinita considerando-se o material homogêneo, a forma geométrica do produto como aproximação a uma placa plana e o coeficiente de difusão constante (Brooker et al., 1992).

$$
R U=\frac{X_{t}-X^{*}}{X_{i}-X^{*}}=\frac{8}{\pi^{2}} \sum_{n=0}^{\infty} \frac{1}{(2 n+1)^{2}} \exp \left[-\frac{D \pi^{2}(2 n+1)^{2} t}{4 L_{0}^{2}}\right]
$$

Em que: $t=$ tempo [s], $D=$ coeficiente de difusividade $\left[\mathrm{m}^{2} \mathrm{~s}^{-1}\right], \mathrm{n}=$ número de termos, $L o=$ metade da espessura da placa plana $[\mathrm{m}]$.

O coeficiente de difusividade pode ser aumentado com o aumento da temperatura, tornando possível aplicar a equação de Arrhenius (Equação 5) para descrever essa relação. Para avaliar a influência da temperatura no coeficiente de difusão efetivo utilizou-se a equação de Arrhenius.

$$
D=D_{0} \exp \left[-\frac{E_{a}}{R T}\right]
$$

Em que: $E a=$ energia de ativação $\left[\mathrm{J} \mathrm{mol}^{-1}\right], D o=$ fator pré-exponencial $\left[\mathrm{m}^{2} \cdot \mathrm{s}^{-1}\right], T=$ temperatura $[\mathrm{k}] \mathrm{e}$ $R=$ constante universal dos gases $\left[\mathrm{mol}^{-1} \mathrm{~K}^{-1}\right]$.

\section{RESULTADOS E DISCUSSÕES}

O teor médio de umidade para as folhas de Pariri in natura foi de $69,66 \pm 2,05 \%$. As curvas que descrevem o comportamento da secagem das folhas da Arrabidaea chica (Humb. \& Bonpl) B. Verlot, nas diferentes condições de temperaturas são expressas na Figura 1. 
Figura 1- Variações da razão de umidade em função do tempo nas diferentes temperaturas para (a) secador de bandejas (b) infravermelho
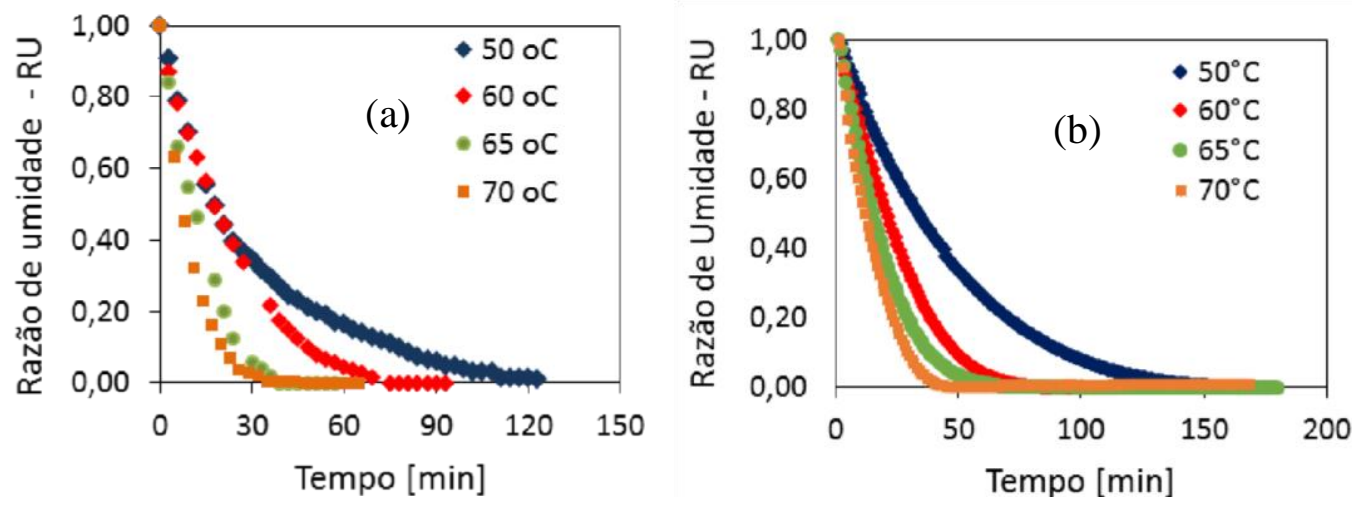

Observa-se pela Figura 1 a influência da temperatura do ar na cinética de secagem Como previsto, o tempo de secagem decresce com o aumento da temperatura do ar. Para se alcançar o equilíbrio no secador de bandejas, os tempos necessários foram de 35, 38, 75 e $110 \mathrm{~min}$, para as temperaturas de $70,60,65$ e $50{ }^{\circ} \mathrm{C}$, respectivamente. No infravermelho os tempos de secagem foram de 46, 70, 87 e 143 min, para as mesmas temperaturas. Ressalta-se que massas distintas foram utilizadas nos secadores.

Apresenta-se, na Tabela 1, o resumo do ajuste dos modelos por meio de regressão não linear aos dados experimentais de secagem de folhas de Pariri em secador de bandejas e infravermelho, considerando-se as diferentes temperaturas do ar de secagem. Dentre os modelos avaliados, a equação de Midilli et al. foi a que melhor se ajustou aos dados de razão de umidade, fato esse evidenciado pela magnitude do $\mathrm{R}^{2}$. Tal fato ocorre para ambos os secadores. Comumente, o modelo de Midilli et at. apresenta um bom ajuste aos dados de secagem de folhas de plantas medicinais, aromáticas ou condimentares, sendo que, este também foi o modelo de melhor ajuste aos dados experimentais de secagem de folhas de Juá (Sousa, 2015), para o estudo de secagem de folhas de manjericão (REIS et al., 2012), folhas de capimlimão (Martinazzo, 2007).

Nos estágios inicias do processo de secagem de plantas medicinais ocorrem rápida perda de água, caracterizando uma curva mais acintosa, justificando o ajuste do modelo de Midilli et al. aos dados experimentais (Silva, 2014). Entretanto, segundo Radünz et al. (2011) o ajuste do modelo matemático depende das características de cada espécie vegetal sendo necessária a realização de estudos individuais para as diversas espécies.

Os valores da difusividade efetiva obtidos para folhas de Pariri em diferentes temperaturas do ar de secagem são explicitados na Figura 2. Nota-se que a difusividade é diretamente proporcional à temperatura do ar de secagem mostrando, assim, uma redução na resistência interna de secagem com o aumento da temperatura. Akpinar (2006) obteve valores entre 4,53 x $10^{-12}$ a 7,04 x $10^{-12} \mathrm{~m}^{2} \mathrm{~s}^{-1}$ para as espécies menta, salsinha e manjericão. Tem-se que as energias de ativação calculadas a partir das retas obtidas na Figura 2, foram de 58,86 kJ $\mathrm{mol}^{1}$ e $39,26 \mathrm{~kJ} \mathrm{~mol}^{-1}$ para os secadores de bandeja e infravermelho, respectivamente. Goneli et al. (2007) relatam que em processos de secagem quanto menor a energia de ativação maior a difusividade de água no produto. 
Tabela 1 - Parâmetros dos modelos de cinética de secagem em secador de bandejas.

\begin{tabular}{|c|c|c|c|c|c|c|c|c|c|}
\hline Modelo & $\mathrm{T}\left({ }^{\circ} \mathrm{C}\right)$ & \multicolumn{2}{|c|}{$50^{\circ} \mathrm{C}$} & \multicolumn{2}{|c|}{$60^{\circ} \mathrm{C}$} & \multicolumn{2}{|c|}{$65^{\circ} \mathrm{C}$} & \multicolumn{2}{|c|}{$70^{\circ} \mathrm{C}$} \\
\hline \multirow{4}{*}{ Page } & parâmetros & $\begin{array}{l}\text { Bandej } \\
\text { as }\end{array}$ & Infrav. & Bandejas & Infrav. & Bandejas & Infrav. & Bandejas & Infrav. \\
\hline & $k\left(\min ^{-1}\right)$ & 0,051 & 0,010 & 0,026 & 0,012 & 0,040 & 0,020 & 0,072 & 0,026 \\
\hline & $N$ & 0,888 & 1,183 & 1,153 & 1,331 & 1,227 & 1,294 & 1,151 & 1,314 \\
\hline & $R^{2}$ & 0,996 & 0,998 & 0,997 & 0,998 & 0,996 & 0,999 & 0,999 & 0,996 \\
\hline \multirow{5}{*}{$\begin{array}{l}\text { Midilli et } \\
\text { al. }\end{array}$} & $A$ & 1,025 & 1,000 & 0,973 & 0,973 & 0,987 & 1,010 & 0,999 & 1,009 \\
\hline & $k\left(\min ^{-1}\right)$ & 0,067 & 0,012 & 0,025 & 0,010 & 0,041 & 0,022 & 0,072 & 0,027 \\
\hline & $N$ & 0,809 & 1,136 & 1,143 & 1,371 & 1,206 & 1,273 & 1,147 & 1,304 \\
\hline & $b\left(\min ^{-1}\right)$ & $-2,0.10^{-4}$ & $-1,24.10^{-4}$ & $-2,8.10^{-4}$ & $-6,52 \cdot 10^{-5}$ & $-3,5.10^{-4}$ & $-9,00 \cdot 10^{-7}$ & $-3,7.10^{-5}$ & $5,95.10^{-5}$ \\
\hline & $R^{2}$ & 0,998 & 0,999 & 0,999 & 0,999 & 0,997 & 0,999 & 0,999 & 0,997 \\
\hline \multirow{3}{*}{$\begin{array}{l}\text { Henderson } \\
\text { e Pabis }\end{array}$} & $A$ & 0,958 & 1,077 & 1,031 & 1,127 & 1,0461 & 1,137 & 1,022 & 1,153 \\
\hline & $k\left(\min ^{-1}\right)$ & 0,032 & 0,023 & 0,044 & 0,044 & 0,0791 & 0,058 & 0,107 & 0,076 \\
\hline & $R^{2}$ & 0,993 & 0,994 & 0,992 & 0,988 & 0,989 & 0,995 & 0,997 & 0,991 \\
\hline \multirow{2}{*}{$\begin{array}{l}\text { Newton e } \\
\text { Lewis }\end{array}$} & $k\left(\min ^{-1}\right)$ & 0,034 & 0,021 & 0,043 & 0,012 & 0,076 & 0,020 & 0,105 & 0,026 \\
\hline & $R^{2}$ & 0,991 & 0,990 & 0,991 & 1,331 & 0,987 & 1,294 & 0,996 & 1,314 \\
\hline
\end{tabular}

Figura 2- Representação de Arrhenius para a relação entre a difusividade efetiva das secagens $1 / \mathrm{T}[\mathrm{K}]$

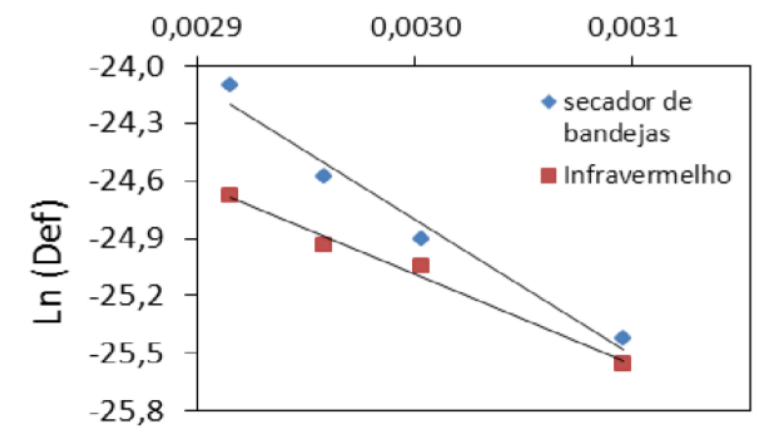

\section{CONSIDERAÇÕES FINAIS}

Do estudo concluiu-se que: a) o comportamento das curvas de secagem das folhas de Pariri foi semelhante ao da maioria dos produtos agrícolas; b) o modelo matemático de secagem proposto por Midilli et al. foi o que melhor se ajustou aos dados experimentais; c) qualitativamente verifica-se que a cor das folhas de Pariri sofreu alterações devido à temperatura de secagem tornando-se mais escuras devido à perda de clorofila.

\section{REFERÊNCIAS}

BROOKER, D. B.; BAKKER-ARKEMA, F. W.; HALL, C. W. Drying and storage of grains and oilseeds. Westport: AVI, 1992. $450 \mathrm{p}$.

CELESTINO, S. M. C. Princípios da Secagem de Alimentos. - Planaltina, DF: Embrapa Cerrados, 2010, 51 p. - (Documentos/ Embrapa Cerrados, ISSN 1517- 5111, ISSN online $2176-5081 ; 276)$. 
CORRÊA, Ricardo Monteiro. Rendimento de óleo essencial e caracterização organoléptica de folhas de assa-peixe submetidas a diferentes métodos de secagem. 2004. Ciência e Agrotecnologia vol.28, n.2, Lavras Mar./Apr. 2004.

FERREIRA, Margarete Martins Pereira. Desenvolvimento, caracterização e secagem de sementes com cobertura artificial. 2003. 265 f. Monografia (Especialização) - Curso de Engenharia Química, Centro Tecnológico de Ciências Exatas, Universidade Federal de São Carlos, São Carlos- Sp, 2004. Cap. 4.

GONELI, A. L.; CORRÊA, P. C.; Resende, O.; Reis Neto, S. A. Estudo da difusão de umidade em grãos de trigo durante a secagem. Ciência e Tecnologia de Alimentos, v.27, p.135-140, 2007.

HARBORNE, J.B. Comparative biochemistry of the flavonoids-VI. : Flavonoid patterns in the bignoniaceae and the gesneriaceae. 1967. Phytochemistry vol 6, Issue 12, December 1967, Pages 1643-1651.

JUNQUEIRA, Maria Heloisa. Modelagem estatística do processo de secagem de folhas de espinheira-santa (Maytenus ilicifolia Mart. ex Reiss). 2016. 15 f. Dissertação (Mestrado) - Curso de Engenharia Agrícola, Universidade Federal da Grande Dourados, Dourados Ms, 2016.

MARTINAZZO A. P., CORRÊA P.C., OSVALDO RESENDE E EVANDRO DE C. M. Análise e descrição matemática da cinética de secagem de folhas de capim-limão. Revista Brasileira de Engenharia Agrícola e Ambiental v.11, n.3, p.301-306, 2007.

PAULETTI, Patrícia Mendonça et al. Constituintes químicos de Arrabidaea samydoides (Bignoniaceae). 2003. Química Nova v.26, n.5, São Paulo set./out. 2003.

PEREIRA, Aline Gabriela Martins. Influência da intensidade de radiação infravermelha na secagem do sistema semente-gel. In: VIII Congresso Brasileiro de Engenharia Química em Iniciação Científica, 8., 2009, Uberlândia. Online. Uberlândia: Galoá, 2009. v. 8

RADÜNZ1, Lauri Lourenço et al. AVALIAÇÃO DA CINÉTICA DE SECAGEM DE CARQUEJA. 2011. REVENG vol.19, n. 1, p.19-27, Engenharia na agricultura, 2011. Viçosa - mg

REIS, Renata C. dos et al. Cinética de secagem de folhas de manjericão (Ocimum basilicum L.) via infravermelho. Revista Brasileira de Engenharia Agrícola e Ambiental, Campina Grande - Pb, v. 16, n. 12, p.1346-1352, 24 dez. 2011.

SALAGNAC, P. et al. Numerical modeling of heat and mass transfer in porous medium during combined hot air, infrared and microwaves drying. 2004. International Journal of Heat and Mass Transfer vol 47, Issues 19-20, September 2004, Pages 4479-4489.

SILVA,L. A. et al. Cinética de secagem e difusividade efetiva em folhas de jenipapo (Genipa americana L.). 2014. Rev. Bras. de Plantas Med. 2015, vol.17, n.4, suppl.2, p.953-963.

SOUSA, F.C. Predição de modelos sobre a cinética de secagem de folhas de Ziziphus joazeiro Mart. Revista Brasileira de Plantas Medicinais, Campina Grande - Pb, v. 17, n. 2, 02 jul. 2015.

TAFFARELlO, Denise. Extrato de Arrabidaea chica (Humb. \& Bonpl) Verlot obtidos por processos biotecnológicos: otimização da extração e avaliação farmacológica. 2008. 191 f. Dissertação (Mestrado em Biotecnologia)- Instituto de Ciências Biomédicas, Universidade de São Paulo, São Paulo, 2009. 